\title{
The Effect of Cranial Nerve Stimulation on Swallowing: A Systematic Review
}

\author{
Michelle G. M. H. Florie ${ }^{1,2}$ - Walmari Pilz ${ }^{1,3} \cdot$ Remco H. Dijkman ${ }^{1} \cdot$ Bernd Kremer $^{1,2} \cdot$ Anke Wiersma $^{1}$. \\ Bjorn Winkens ${ }^{4} \cdot$ Laura W. J. Baijens ${ }^{1,2}$
}

Received: 5 January 2020 / Accepted: 2 May 2020 / Published online: 14 May 2020

(c) The Author(s) 2020

\begin{abstract}
This systematic review summarizes published studies on the effect of cranial nerve stimulation (CNS) on swallowing and determines the level of evidence of the included studies to guide the development of future research on new treatment strategies for oropharyngeal dysphagia (OD) using CNS. Studies published between January 1990 and October 2019 were found via a systematic comprehensive electronic database search using PubMed, Embase, and the Cochrane Library. Two independent reviewers screened all articles based on the title and abstract using strict inclusion criteria. They independently screened the full text of this initial set of articles. The level of evidence of the included studies was assessed independently by the two reviewers using the A-B-C rating scale. In total, 3267 articles were found in the databases. In the majority of these studies, CNS was used for treatment-resistant depression or intractable epilepsy. Finally, twenty-eight studies were included; seven studies on treatment of depression, thirteen on epilepsy, and eight on heterogeneous indications. Of these, eight studies reported the effects of CNS on swallowing and in 20 studies the swallowing outcome was described as an adverse reaction. A meta-analysis could not be carried out due to the poor methodological quality and heterogeneity of study designs of the included studies. These preliminary data suggest that specific well-indicated CNS might be effective in reducing OD symptoms in selective patient groups. But it is much too early for conclusive statements on this topic. In conclusion, the results of these studies are encouraging for future research on CNS for OD. However, randomized, double-blind, sham-controlled clinical trials with sufficiently large sample sizes are necessary.
\end{abstract}

Keywords Dysphagia $\cdot$ Deglutition disorder $\cdot$ Swallowing $\cdot$ Cranial nerve $\cdot$ Cranial nerve stimulation

Michelle G. M. H. Florie

michelle.florie@mumc.nl

1 Department of Otorhinolaryngology, Head and Neck Surgery, Maastricht University Medical Center, PO Box 5800, 6202 AZ Maastricht, The Netherlands

2 GROW-School for Oncology and Developmental Biology, Maastricht University Medical Center, Maastricht, The Netherlands

3 School for Mental Health and Neuroscience - MHeNs, Maastricht University Medical Center, Maastricht, The Netherlands

4 Department of Methodology and Statistics, CAPHRI - Care and Public Health Research Institute, Maastricht University, PO Box 616, 6200 MD Maastricht, The Netherlands

\section{Introduction}

Cranial nerves play an important role in swallowing, a complex cognitive, sensorimotor process of moving any bolus from the mouth to the stomach [1,2]. The stages of swallowing can be seen as a complex activity along a spectrum of automaticity, with the esophageal stage being most automatic and the oral stage the least [1]. The nervous system controlling swallowing movements has mammalian brain stem and midbrain patterning control systems (automatic areas) as well as cortical and subcortical volitional areas [2]. But in all stages of swallowing the cranial nerves play a major role in modulating swallowing execution and their integrity is indispensable [3].

The trigeminal nerve (TN), the fifth cranial nerve, controls somatosensation of the face and the anterior two-thirds of the tongue [4, 5]. It provides motor innervation of (1) the mylohyoid muscle and the anterior belly of the digastric 
muscle, which are hyolaryngeal elevators; (2) the masticatory muscles, such as the masseter, temporalis, medial and lateral pterygoid muscles; and (3) the tensor veli palatini muscle. Impairment of the $\mathrm{TN}$ can cause problems in the preparatory oral and oral phase of swallowing due to poor mastication and poor stabilization of the mouth floor. TN dysfunction will also result in a decreased hyolaryngeal excursion during the pharyngeal phase of swallowing due to mylohyoid and anterior belly digastric muscle impairment.

The facial nerve (FN), the seventh cranial nerve, conveys taste sensation of the anterior two-thirds of the tongue [4]. Furthermore, the FN controls motor movement of the muscles of facial expression such as the orbicularis oris and buccinator muscle, both playing a role in lip closure and prevention of oral residue. The FN also provides motor innervation of the stylohyoid muscle and the posterior belly of the digastric muscle. These muscles retract the hyoid bone posterosuperiorly and assist in glossopalatal closure. Innervation of the submandibular and sublingual salivary glands is provided by the FN too. Impairment of the FN can result in decreased taste perception, poor bolus formation during the preparatory oral phase, anterior bolus spilling, postswallow oral residue, and dry mouth.

The glossopharyngeal nerve (GN), the ninth cranial nerve, controls somatosensation of the posterior one-third of the tongue and of the mucosa of the soft palate and the upper pharyngeal tract [4]. The autonomic innervation of the parotid gland is also provided by the GN. The motor innervation of the GN is the innervation of the stylopharyngeus muscle, which is a laryngeal elevator assisting in the opening of the upper esophageal sphincter (UES). The GN also plays a key role in soft palate elevation as the salpingopharyngeus muscle is innervated by the GN and this muscle fuses inferiorly with the fibers of palatopharyngeus muscle. GN dysfunction can result in impaired pharyngeal bolus transport and impaired UES opening resulting in postswallow pharyngeal pooling.

The vagal nerve $(\mathrm{VN})$, the tenth cranial nerve, provides both motor and sensory innervation, and plays an important role in the pharyngeal phase of swallowing $[4,6]$. The motor fibers of the $\mathrm{VN}$ innervate all striated muscles of the larynx and pharynx, except the stylopharyngeus muscle and the tensor veli palatini muscle, which are innervated by the GN and by the TN, respectively. The pharyngeal branches of the VN innervate the levator veli palatini, salpingopharyngeus, palatopharyngeus, palatoglossus, and the uvular muscle. The external superior laryngeal nerve (SLN) supplies the motor innervation of the cricothyroid muscle. The recurrent laryngeal nerve (RLN) is responsible for the motor innervation of all intrinsic laryngeal muscles except for the cricothyroid muscle. Various branches of the VN, like the RLN and the internal branch of the SLN (ISLN), provide mucosal sensory innervation of the pharynx, larynx, and proximal trachea.
Impairment of the VN can cause poor velopharyngeal seal and nasal reflux, weak pharyngeal contraction, reduced vocal fold adduction resulting in dysphonia and poor cough effectiveness, impaired UES opening — postswallow pharyngeal pooling, and silent aspiration.

The hypoglossal nerve (HN), the twelfth cranial nerve, innervates all intrinsic and extrinsic tongue muscles, except for the palatoglossus muscle (VN). The HN is a nerve with exclusively motor function controlling all movements of the tongue. Besides dysarthria, impairment of the $\mathrm{HN}$ can cause problems with oral control of the bolus, bolus propulsion due to poor lingual pressure and driving forces resulting in premature posterior spill of the bolus to the pharynx, postswallow oral residue, etc.

The ansa cervicalis (AC), the connection between the cervical plexus $(\mathrm{C} 1, \mathrm{C} 2)$ and the $\mathrm{HN}$, is a loop of nerves, which innervates the omohyoid muscle's superior belly as well as the superior part of the sternothyroid and the sternohyoid muscles $[3,7]$. Activation of these muscles initiates hyolaryngeal elevation. Furthermore, the $\mathrm{AC}$ also seems to assist in airway protection by compression of the quadrangular membrane thereby assisting in closure of the laryngeal inlet in collaboration with the TN and VN [8]. Function loss of the AC can cause an impaired UES opening, resulting in postswallow pharyngeal pooling.

Cranial nerve palsy is characterized by a decreased or complete loss of function of one or more cranial nerves. The etiology may be congenital or acquired. Multiple cranial neuropathies of cranial nerves involved in swallowing are common, particularly in lesions arising from tumors, trauma, head-and-neck surgery, impaired blood flow, and infections. For example, cranial nerves FN, VN, HN, and AC are especially at risk during head-and-neck cancer surgery [9]. Tumor extension into a cranial nerve often results in sacrificing this nerve during a radical or modified radical neck dissection. Also in thyroid gland surgery cranial nerves may be at risk in particular the VN-RLN. If multiple cranial nerve palsy occurs for no apparent reason, abnormalities in the base of the skull and brain should be considered. Also paraneoplastic symptoms or infections caused by neurotropic viruses such as those of the herpes group should be part of the differential diagnoses. Finally, cranial nerve palsy may be the result of a stroke [8]. Bilateral cranial nerve palsy or asymmetrically neuromuscular representation and the potential recovery depend on the size and site of the lesion in the brain.

During the past two decades, several studies on cranial nerve stimulation (CNS) have been published. CNS is a medical treatment for symptoms of various diseases, in which electrical signals stimulate cranial nerves in order to modulate the activity of targeted brain regions or to modulate the action of peripheral structures. Currently, there are two types of CNS that are used in daily clinical practice, 
namely vagal nerve stimulation (VNS) and hypoglossal nerve stimulation (HNS).

CNS has been applied for several diseases and/or syndromes such as epilepsy, obstructive sleep apnea syndrome, obesity, neuropsychiatric disorders (depression, obsessive compulsive disorder, panic disorder, pain disorder, post-traumatic stress disorder, etc.), and asthma exacerbations [10-20]. Few studies described the effect of CNS on swallowing and, consequently, the overall effect of this intervention on swallowing remains unclear.

Various treatments for oropharyngeal dysphagia (OD) have been described in the literature, including swallowing exercises that aim to change swallow physiology through targeting strength and/or range of movement of muscles and/or neuroplasticity in the brain, surgical interventions (UES interventions), bolus modification (modified texture diet), neuromuscular electrical stimulation (E-stimulation etc.), transcranial magnetic stimulation, postural and airway protective strategies/maneuvers that facilitate swallowing, pharmacological interventions, etc. [21-24]. CNS has only been scarcely investigated as a treatment for OD and evidence of its effectiveness is part of the subsequent systematic review in the present study. The systematic review was build based on the following question: Is there any scientific evidence that CNS can be used as a novel treatment for OD in specific patient populations?

The aim of this systematic literature review was to present an overview of the studies on the effect of CNS on swallowing and to determine the level of evidence of these studies to guide the development of future research on new treatment strategies for OD using CNS.

\section{Methods}

\section{Identification and Selection of Studies}

This review was conducted following the Cochrane Collaboration criteria for systematic reviews [25]. The literature search using the electronic databases Embase, PubMed, and the Cochrane library was carried out on November 1, 2019 by two independent investigators. Search terms were related to dysphagia and to CNS. The search strategy is presented in Table 1. The search was limited to articles published between January 1990 and October 2019. Studies were included if they described any effect of CNS on the oropharyngeal swallowing physiology even if the effect was reported as an adverse reaction (AR). The in- and exclusion

Table 1 Systematic syntax

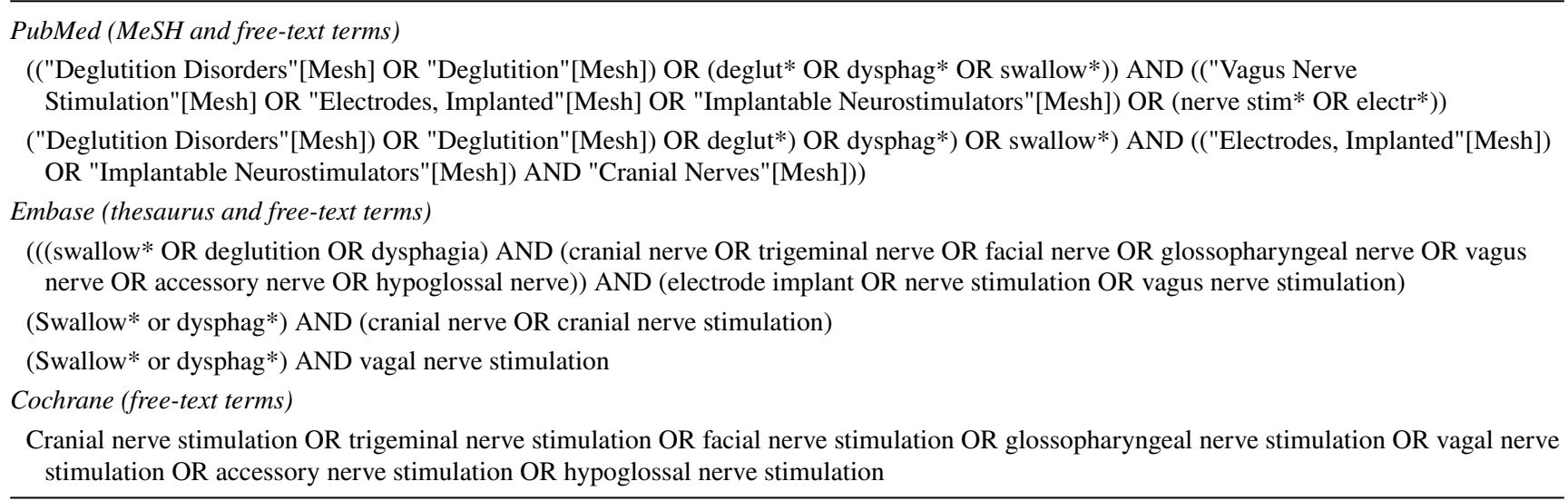

*Truncation of search terms to broaden the search

Table 2 In- and exclusion criteria

\author{
Inclusion criteria \\ Studies describing swallowing function and/or OD associated with CNS \\ Studies describing OD and/or aspiration even as an adverse reaction of CNS \\ Studies on human subjects \\ Exclusion criteria \\ Studies including other forms of stimulation such as transcutaneous, intrapharyngeal, or deep brain \\ stimulation \\ Studies on CNS that did not report swallowing function or OD as outcome variable or adverse reaction \\ Animal studies
}

$C N S$ cranial nerve stimulation, $O D$ oropharyngeal dysphagia 
criteria are listed in Table 2. Peer-reviewed journal articles written in the English, German, Portuguese, Spanish, French, or Dutch language were included in the search. Studies involving experiments on animals were excluded. Articles were also excluded if swallowing outcomes were not presented in the results. Two independent reviewers performed the first selection by screening all articles based on title and abstract. During the next step they independently screened the full text of the selected set of articles. Finally, the reference lists of the selected articles were screened for additional literature. All studies reporting on swallowing and CNS and meeting the in- and exclusion criteria were included. The level of agreement between the two reviewers for eligibility after full-text screening was obtained using percentage of agreement. Figure 1 comprises a flow diagram showing the article selection according to Preferred Reporting Items for Systematic Reviews and Meta-Analyses (PRISMA) [26].

\section{Data Analysis and Assessment of the Level of Evidence}

The level of evidence of the included studies was assessed using the A-B-C rating scale by Siwek et al. [27]. This assessment was carried out by both reviewers independently, blinded to each other's results. This design-specific

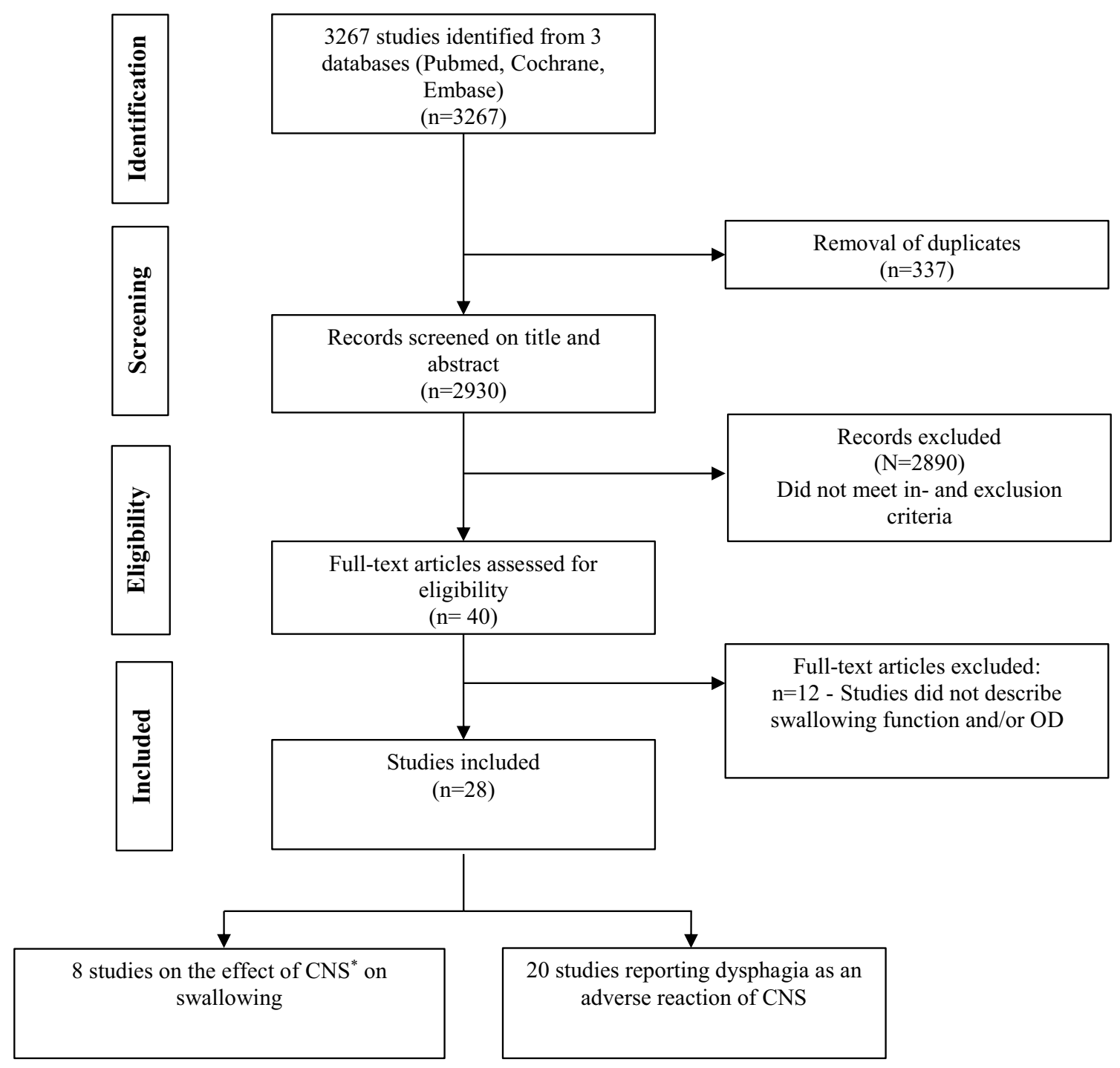

${ }^{*} \mathrm{CNS}=$ cranial nerve stimulation

Fig. 1 PRISMA study selection 
tool was chosen because the methodological quality of the majority of the included studies was not sufficient to carry out a validated critical appraisal assessment. According to the $\mathrm{A}-\mathrm{B}-\mathrm{C}$ rating scale, level A refers to high-quality randomized controlled trials and meta-analyses, level B refers to well-designed, non-randomized clinical trials, and level C refers to consensus or expert opinion. The level of agreement between the two reviewers for the A-B-C rating scale was obtained using percentage of agreement.

Data extraction included the following variables: sample size, indication for stimulation, cranial nerve number, type of nerve stimulator, stimulation parameters, measurement tool and outcome parameters, authors' conclusion, and the number of OD-related ARs.

\section{Results}

\section{General Results and Level of Evidence}

A total of 2930 articles were identified after removing duplicates using EndNote X7.5 (Clarivate Analytics, Philadelphia, Pennsylvania) (Fig. 1). The percentage of agreement between the two reviewers for the first selection based on title and abstract was $68 \%$. If agreement was not reached based on the title and abstract, the full text of the article was screened. The percentage of agreement between the two reviewers for eligibility after full text screening was $100 \%$. Finally, 28 articles were included for subsequent assessment of the level of evidence (Table 3). The level of agreement between the two reviewers using the $\mathrm{A}-\mathrm{B}-\mathrm{C}$ rating scale was $100 \%$. All the included articles were written in English. All eight studies on the effect of CNS on swallowing (Table 3) met the criteria of level C-expert opinion. Thus, a meta-analysis of the included studies was not carried out as the studies did not have sufficient methodological quality to warrant doing so. More specific, the majority of the studies presented serious methodological shortcomings (e.g., no clear description of the subjects selection criteria; little or incomplete information about diagnostic tools and protocol; no interpretation of the test results). Subsequently, a narrative descriptive review of the systematically included studies was performed. Table 3 provides an overview of the eight studies describing the effect of CNS on swallowing. In this table, the sample size, indication for stimulation, cranial nerve number, type of nerve stimulator, stimulation parameters, measurement tool and outcome parameters, and authors' conclusion are summarized.

Table 4 provides an overview of the twenty studies that described OD as an AR of CNS. In these studies, the indication for stimulation did not target swallowing function. The following data were extracted from the studies: type of nerve stimulator, stimulation parameters, indication for stimulation, and the number of OD-related ARs. In these studies, ARs such as OD were reported based on patient interview or via Coding Symbols for Thesaurus of Adverse Reaction Terms (COSTART) [28].

\subsection{Summary of Studies}

The studies in Table 3 were presented according to the cranial nerve number stimulated (from low-to-high): VN (four studies), ISLN (one study), RLN (two studies), and HN (one study).

\section{Vagal Nerve Stimulation}

Lundgren et al. primarily studied swallowing in seven children, treated for intractable epilepsy with a VN stimulator using videoradiography with barium swallowing [29]. Three stimulation conditions were compared to observe the effects of VNS on swallowing ("MAX"; "in use"; "off”). During almost continuous VNS ("MAX") an increased penetrationaspiration score was observed in two of the seven children. In one patient, this increased score during "MAX" was observed compared to the "in use" setting and in the other patient it was observed during "MAX" compared to the "off" setting. However, no significant difference in penetrationaspiration score was found between the "in use" versus "off" condition in all children.

Schallert et al. studied eight children with pharmacoresistant epilepsy using a left-sided-VN stimulator to determine whether stimulation could affect swallowing [30]. They concluded that stimulation of the left $\mathrm{VN}$, under the conditions used to treat epilepsy, did not cause aspiration during barium swallow videoradiography, although one patient showed laryngeal penetration during "therapeutic stimulation" (condition "on") versus no laryngeal penetration during no stimulation (condition "off").

Zalvan et al. retrospectively reported ARs of VNS on swallowing in four patients with intractable epilepsy in a case series study [31]. Swallowing was evaluated using patient interview and fiberoptic endoscopic evaluation of the laryngeal function. Symptoms of OD, such as gagging or coughing while drinking or feeding difficulties during stimulation (condition "on"), were reported by three of the four subjects. Furthermore, the authors reported that these ODrelated symptoms persisted in one subject despite switching off the stimulator (condition "off"). After adjustment of the VNS parameters, two subjects did not show OD-related symptoms anymore.

Marrosu et al. investigated the effect of VNS on swallowing in three males affected by multiple sclerosis (MS) presenting postural cerebellar tremor (PCT) and OD [32]. Following VNS OD for thin liquid measured using a swallowing speed test and the PCT improved in all subjects during the 


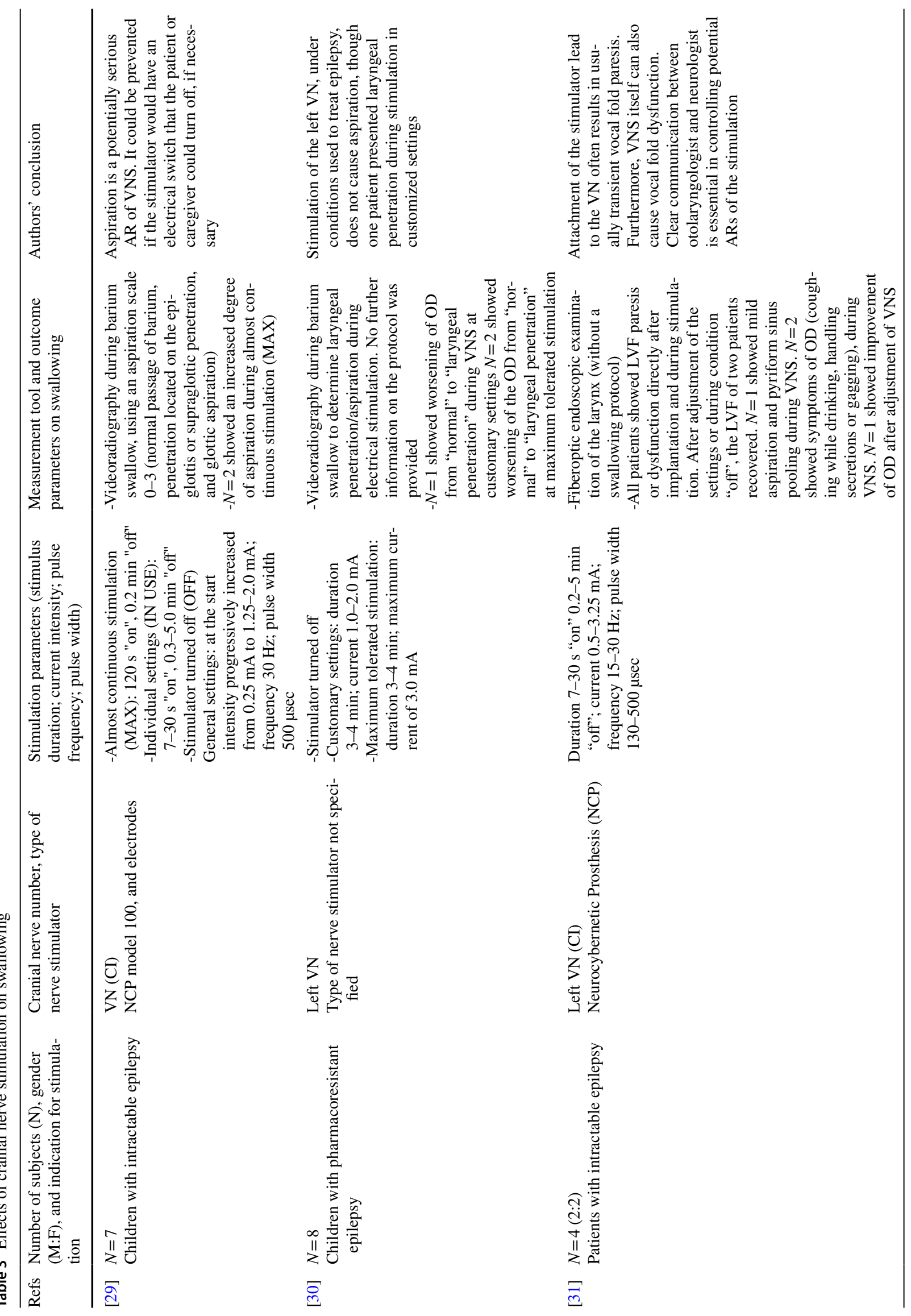




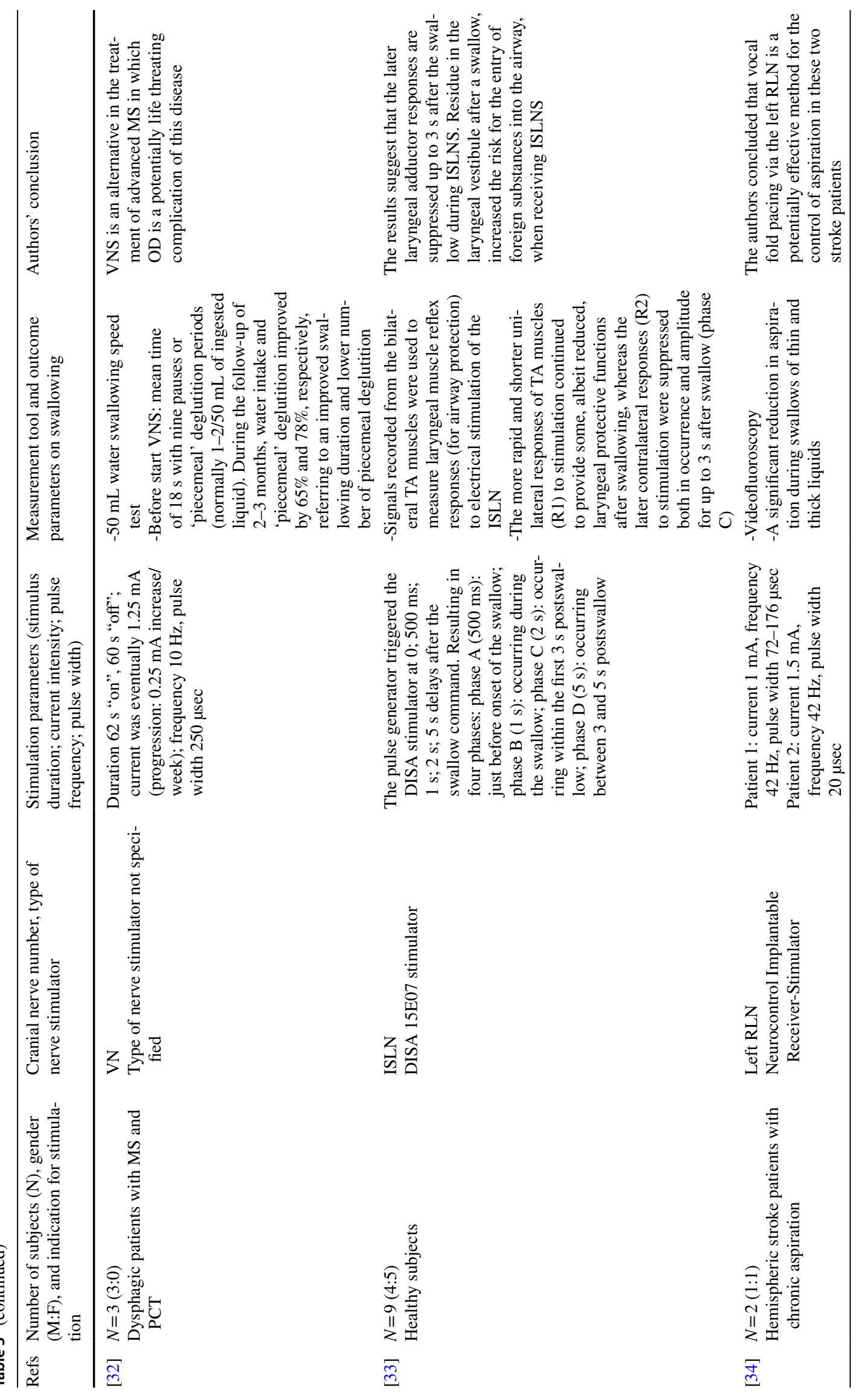




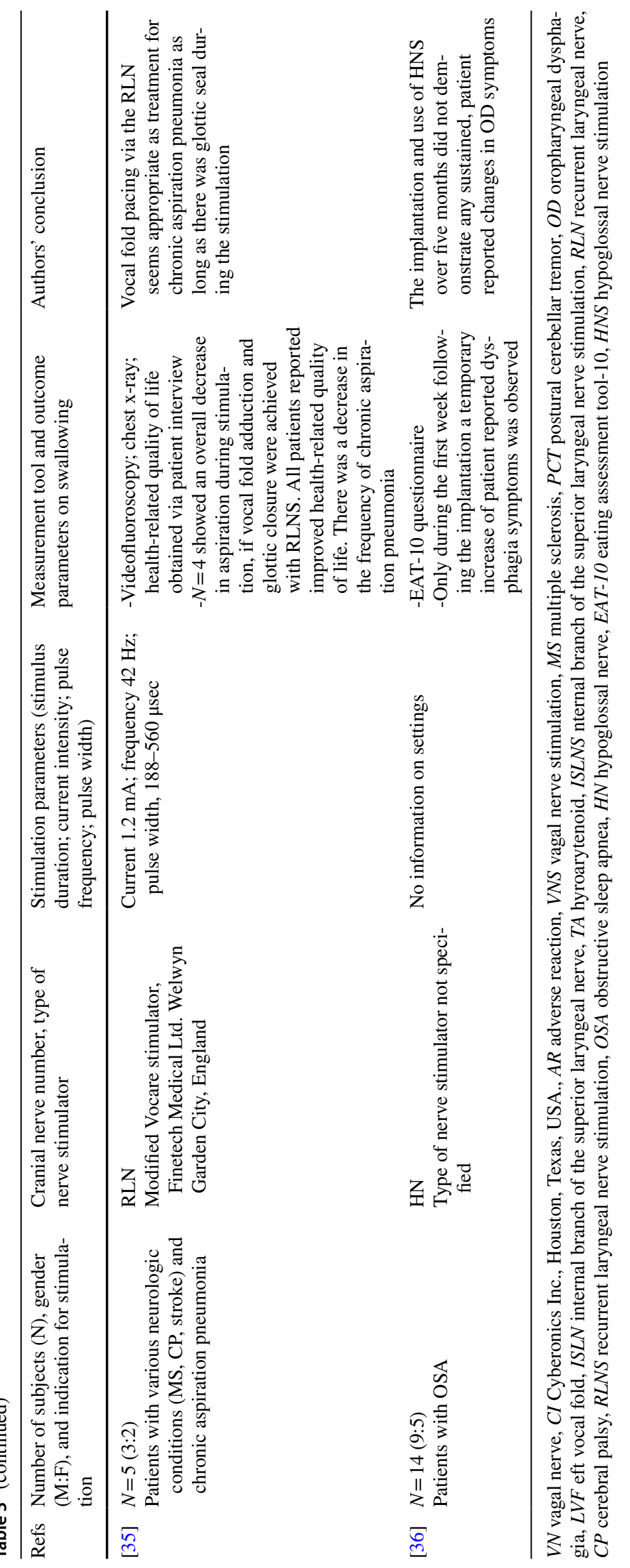




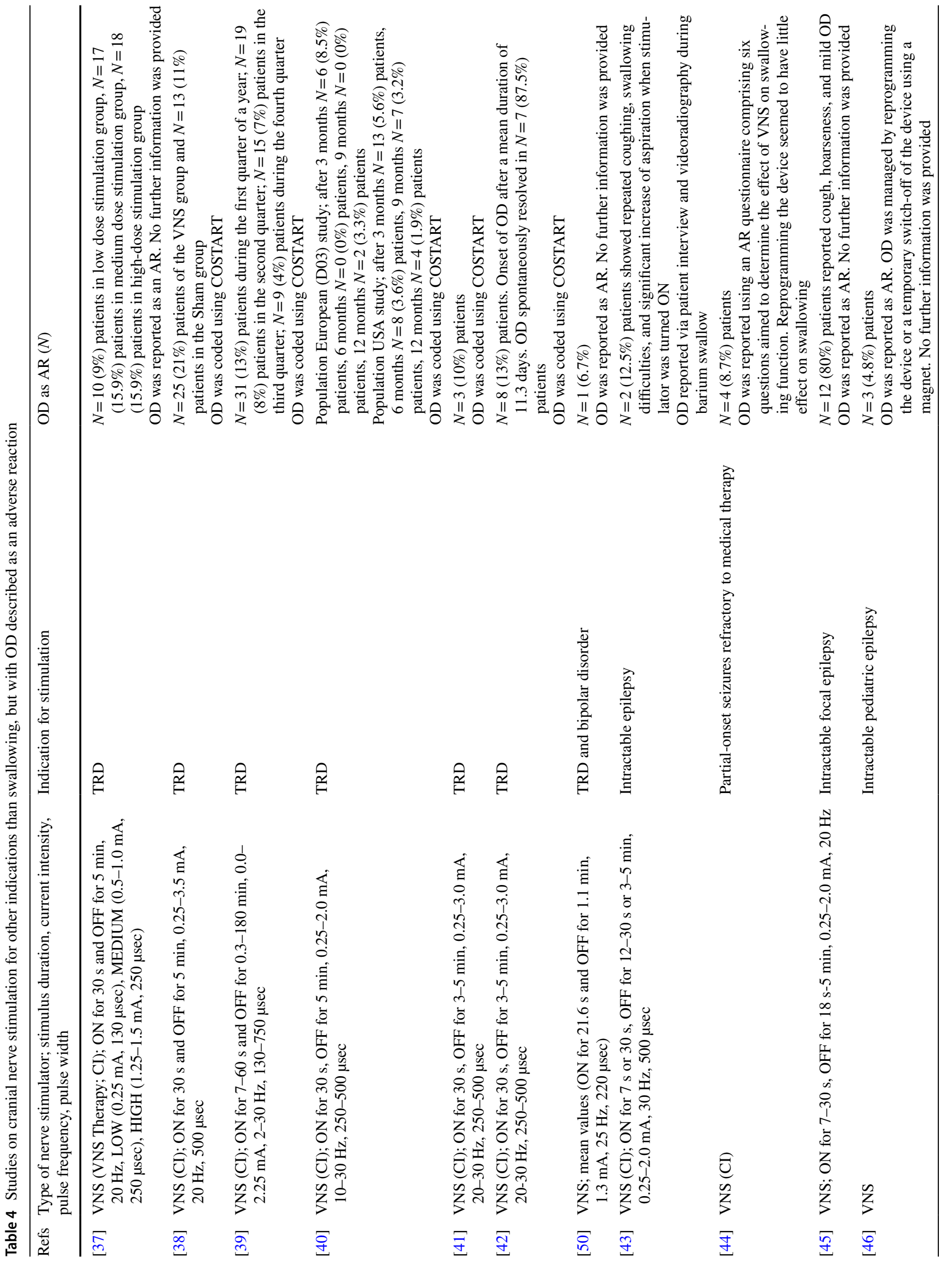




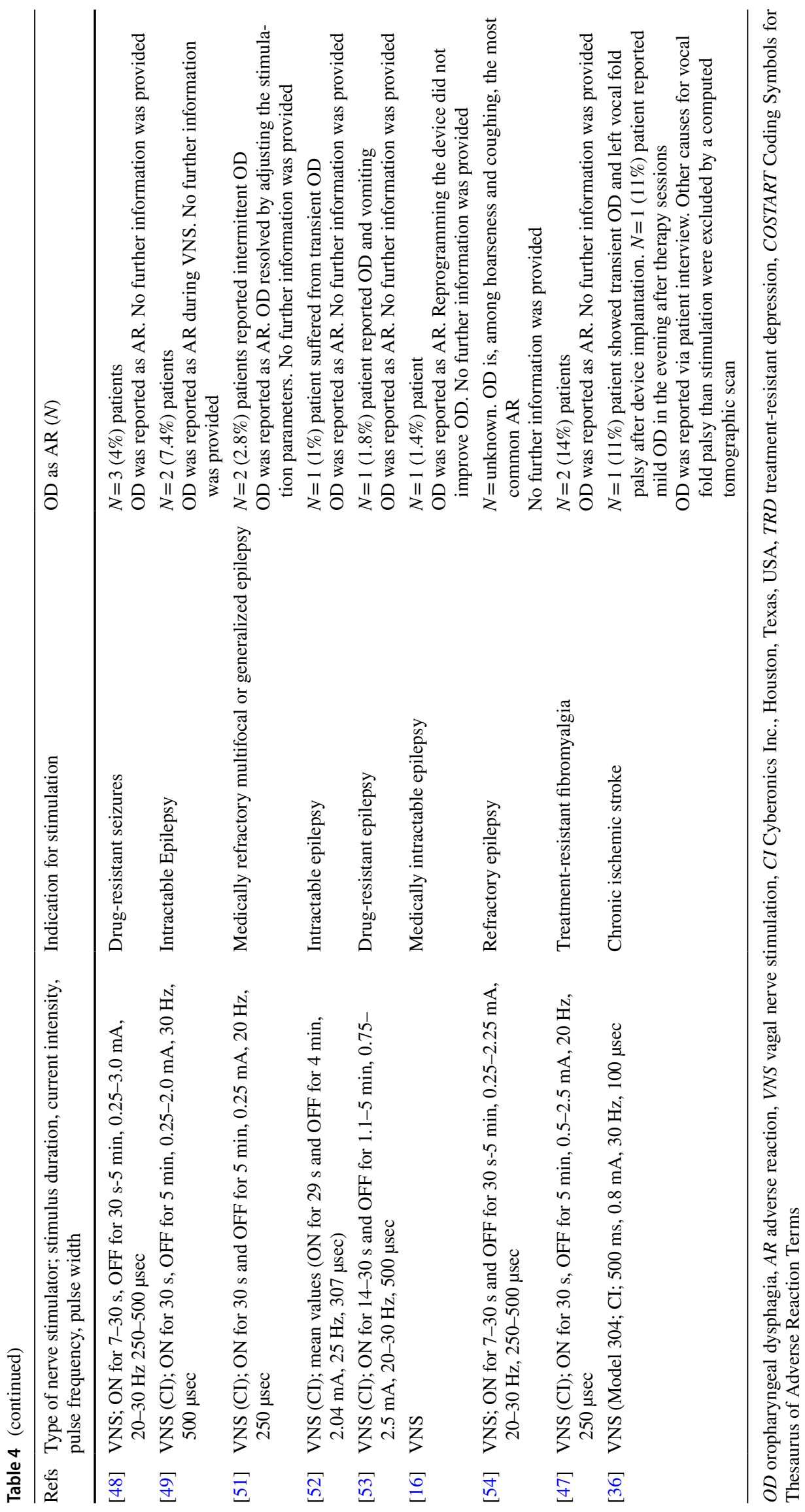


follow-up period of two to three months. Difficulties in swallowing solids failed to improve, although specific information on swallowing outcome variables was not reported.

\section{Internal Branch of the Superior Laryngeal Nerve Stimulation}

Central nervous system suppression of laryngeal adductor responses during swallowing was studied in nine healthy subjects by Barkmeier et al. [33]. They studied the frequency and amplitude of laryngeal adductor responses in the thyroarytenoid (TA) muscle such as the rapid and shorter ipsilateral (R1) responses and later contralateral (R2) responses. The authors used bipolar needle electrodes during electrical stimulation of the ISLN in different phases of volitional swallowing. The results demonstrated a suppression of laryngeal sensorimotor $\mathrm{R} 2$ responses up to three seconds following a swallow command. The R1 response frequency, however, was not affected during all phases of swallowing. These results suggested that stimulation of the ISLN is suppressing the R2 laryngeal protective function (adductor responses), putting people at risk for aspiration. However, the $\mathrm{R} 1$ rapid protective sensorimotor response remained intact and could still be triggered during bolus entry into the laryngeal vestibule and penetration up to the level of the vocal folds evoking a cough reflex.

\section{Recurrent Laryngeal Nerve Stimulation}

Broniatowski et al. described the effect of RLN stimulation (RLNS) on aspiration in two stroke patients with a tracheostomy and chronic aspiration [34]. There was a significant reduction in aspiration during videofluoroscopy in these two patients during thin and thick liquid swallows under RLNS. Swallows of puree consistency did not improve during RLNS. The authors concluded that swallowing of thin and thick liquid consistencies was safe as a result of RLNS.

Subsequently, Broniatowski et al. studied the effect of RLNS on aspiration during videofluoroscopy in three additional patients extending their sample size to five patients (including the two patients of their previous study) [35]. The effect of RLNS on health-related quality of life, measured with a patient interview and pneumonia rates was reported in the study. Patients were suffering from stroke, MS, or cerebral palsy. In four of the five patients, the frequency of aspiration decreased using RLNS and their health-related quality of life improved. One stroke patient did not experience any beneficial effect of RLNS on health-related quality of life or on pneumonia rate. The authors concluded that RLNS is a potentially effective method for the reduction of aspiration and that RLNS might have an added value in the prevention of chronic aspiration pneumonia.

\section{Hypoglossal Nerve Stimulation}

Bowen et al. described the effect of HNS on swallowing in patients treated for obstructive sleep apnea (OSA) [36]. During the first week following the implantation of the HN stimulator, a temporary increase of OD symptoms, measured with the EAT-10 questionnaire, was observed, which normalized during the next six months. This might indicate that the transient OD-related symptoms were probably due to postoperative wound healing conditions at the implantation site.

Table 4 presents 20 studies where signs of OD such as impaired swallowing safety (aspiration) or impaired swallowing efficiency (pooling/residue) were described as an AR of CNS. In the majority of these studies, ARs were identified using COSTART, swallow-related questionnaires and/ or physical examination. However, in the vast majority of these studies ARs were reported without any information on the applied AR protocol or any other protocol for data collection. In nearly half of these studies, OD was not specified in terms of onset, severity, duration, or dependency of stimulation parameters. In these studies, the frequency of OD signs and symptoms ranged from 1 to $80 \%$.

\section{Discussion}

Despite the fact that the number of indications for CNS has increased in recent years, the number of studies on the effect of this stimulation on swallowing has remained very limited. The aim of this systematic literature review was to present an overview of the studies on the effect of CNS on swallowing and to determine the level of evidence of these studies to guide the development of future research on new treatment strategies for OD using CNS. The systematic search we conducted for this review generated a limited number of articles on the effect of CNS on swallowing [29-36]. Clear conclusions about the evidence could not be drawn as the majority of the included studies were case studies or case series and the overall sample size was very small. Study size varied from 2 to 14 subjects. Four studies analyzed a population of less than five subjects [31, 32, 34, 35]. None of the studies described statistical analyses. The majority of the studies did not report the diagnostic protocol used to assess the swallowing physiology. The studies were considerably heterogeneous regarding patient populations and outcome parameters. The study populations consisted of children and adults with epilepsy, healthy subjects, patients with various neurologic conditions (MS, CP, and stroke), and patients with OSA. No information was reported on any long-term effects of CNS on swallowing. It can be concluded that all included studies of the present 
review did not clearly describe the methodological study protocol making replication of the studies impossible.

The effect of direct electrical stimulation of the TN, FN, and GN on swallowing, either as an AR during stimulation for other non-swallow indications, has not been reported in the literature yet.

Meanwhile, the effects of VNS and HNS on swallowing were primarily reported as ARs [17, 37-55]. For example, in the treatment of depression, VNS seems to induce OD in up to $10 \%$ of the patients according to the systematic review of Martin et al. [56].

The most frequently used nerve for CNS is the VN. VNS has shown to be a minimally invasive procedure in which the stimulator is generally well tolerated compared to other neurosurgical interventions such as deep brain stimulation, motor cortex stimulation, responsive neurostimulation, or spinal cord stimulation $[10,11,13-20,37-46,48-58]$. The VNS device consists of a pulse generator which is placed subcutaneously in the upper chest below the patient's clavicle and bipolar electrodes are tunneled up to the patient's neck and wrapped around the left VN, above the level of the omohyoid muscle in the neurovascular sheet. Although the therapeutic effects of VNS were extensively studied, the exact therapeutic mechanisms underlying neurostimulation remain unclear [58]. It is known that VNS activates neurons in the basal forebrain and locus coeruleus. This activation evokes a release of acetylcholine and norepinephrine facilitating reorganization of cortical networks and enhancement of neural plasticity $[59,60]$. It has been suggested that VNS in conjunction with muscle movement might improve task-specific plasticity in the motor cortex of rats [61]. Subsequent studies on human subjects showed that VNS in conjunction with rehabilitative muscle training drives large-scale synaptic reorganization in motor control networks following stroke and spinal cord injury, and provides an enduring rehabilitation [62, 63]. Current studies are investigating optimal settings for VNS in rats to identify paradigms that maximize neuroplasticity [64]. The question arises whether VNS could contribute to an improvement of neuroplasticity in case of OD due to neurological disorders?

Usually the VN is stimulated for drug-resistant epilepsy and depression [58]. The potential use of VNS for other indications, such as essential tremor, cognitive deficits in Alzheimer's disease, anxiety disorders, and bulimia has been reported in several studies [65]. Furthermore, the effect of anti-epilepsy VNS on swallowing was described in several studies [29-31]. These studies reported increased signs of OD including aspiration as an AR of VNS. Aspiration disappeared in the majority of patients following adjustment of the stimulation parameters. One study described VNS as a primary treatment for OD in patients with MS showing a beneficial effect of VNS on OD complaints [32]. Currently, the level of evidence of studies on VNS is too poor to support VNS as a treatment for OD.

The HN comes in second place as most frequently stimulated cranial nerve and is usually stimulated as a treatment for OSA [58]. Only one single study described the effect of HNS for OSA on swallowing in non-dysphagic patients. A short-term self-limiting patient reported AR on swallowing occurred immediately following surgical implantation. HNS did not have any long-term ARs on swallowing in this study. Yet, it is known that damage of this nerve usually results in dysarthria and problems in the preparatory oral phase and the oral phase of swallowing [66]. An interesting hypothesis arises if we look at the study by Hadley et al. on the effect of HNS in anesthetized canines. The HNS resulted in an increased hyolaryngeal elevation, which plays an important role in airway protection during swallowing [67]. The remaining question is whether HNS can contribute to swallow safety in patients presenting aspiration.

The current systematic review only identified a single study describing the effects of ISLN stimulation (ISLNS) on volitional swallowing in healthy subjects [33]. The authors concluded that ISLNS resulted in an increased risk of aspiration due to suppression of the protective laryngeal adductor responses. Even isolated peripheral ISLN dysfunction without any lesions in the airway or central nervous system can cause an increased aspiration risk [33]. Relevant additional information about the role of the ISLN in swallowing has been described in the study by Jafari et al. They reported that bilateral anesthetic blockage of the ISLN with $0.5 \%$ bupivacaine has a profound effect on swallowing in all twentyone healthy subjects [68]. These healthy subjects showed an increased effort to initiate swallowing and $25 \%$ of them showed penetration or aspiration during videofluoroscopy with liquid barium. Further studies on the effect of ISLNS in dysphagic patients are needed.

Two studies evaluated the effect of RLNS on OD in stroke, MS, and CP patients suffering from chronic aspiration pneumonia [34, 35]. The authors concluded that RLNS seems to improve the glottal seal which might be a potentially effective method to prevent aspiration in these patients. But here too, due to methodological shortcomings the scientific evidence of these studies is so weak that RLNS cannot be recommended as a treatment for OD. Swallowing is a very complicated neurogenic activity that is elicited through a collaboration between different integrated neural pathways in the central and peripheral nervous systems [8]. Miller et al. described that stimulation of specific cranial nerve nuclei in the brainstem did not result in a pharyngeal swallow [69]. However, it remains an interesting question whether stimulation of cranial nerves in their course or at the level of their nuclei in the brainstem can bring about a change that may in the long term be relevant in the context of treatment of certain OD phenotypes. Overall, the research 
question whether there is a beneficial effect of CNS on swallowing remains unanswered.

\section{Limitations of the Review}

The present review has some limitations with respect to the search strategy and data analysis. The systematic search generated a low number of articles on the effect of CNS on swallowing in human subjects. One reason for this low number may be the inconsistent terminology used in this research topic; as a result, it is possible that some eligible studies were missed despite the extended free-text search (Table 1). The systematic search syntax and the analysis of the level of evidence were carried out according to the PRISMA statement. However, the study designs were of such poor methodological quality that a subsequent meta-analysis or qualitative analysis using a validated critical appraisal tool could not be performed. The steps following the analysis of the level of evidence have to be classified under narrative or traditional literature review with a comprehensive, critical, and objective analysis of the current knowledge on this topic. The gray literature was not included because it generally lacks strict bibliographic control, meaning that basic information such as author, publication date, or publishing body may not be easily discerned. Publication bias cannot be ruled out as it is likely that unpublished studies did not find any effects of CNS on swallowing or resulted in severe ARs.

\section{Conclusion}

These preliminary data suggest that specific well-indicated CNS might be effective in reducing OD symptoms in selective patient groups. But it is far too early for conclusive statements on this topic. The reviewers found heterogeneous outcomes and serious methodological.

limitations, which prevented them from pooling data to identify trends that would assist in designing best clinical practice protocols for OD using CNS. However, these first pioneer studies are very important because they confirm the feasibility of CNS as possible treatment option for OD. To date, it is not known whether CNS for OD could have negative effects. Therefore, the risk/benefit ratio should also be included in future studies. In conclusion, the results of these studies are encouraging for future research on CNS for OD. However, randomized, double-blind, sham-controlled clinical trials with adequate sample size are necessary.

\section{Compliance with Ethical Standards}

Conflict of interest The authors declare that they have no conflict of interest.
Open Access This article is licensed under a Creative Commons Attribution 4.0 International License, which permits use, sharing, adaptation, distribution and reproduction in any medium or format, as long as you give appropriate credit to the original author(s) and the source, provide a link to the Creative Commons licence, and indicate if changes were made. The images or other third party material in this article are included in the article's Creative Commons licence, unless indicated otherwise in a credit line to the material. If material is not included in the article's Creative Commons licence and your intended use is not permitted by statutory regulation or exceeds the permitted use, you will need to obtain permission directly from the copyright holder. To view a copy of this licence, visit http://creativecommons.org/licenses/by/4.0/.

\section{References}

1. Rosenbek JC, Jones NJ: Dysphagia in movement disorders. San Diego, CA: Plural Publishing; 2009.

2. Ludlow CL. Central nervous system control of voice and swallowing. J Clin Neurophysiol. 2015;32:294-303.

3. Costa MMB. Neural control of swallowing. Arq Gastroenterol. 2018;55(1):61-75.

4. Jones B. Normal and abnormal swallowing. 2nd ed. New York: Springer; 2002.

5. Sanders RD. The Trigeminal (V) and Facial (VII) cranial nerves: head and face sensation and movement. Psychiatry (Edgmont). 2010;7:13-6.

6. Erman AB, Kejner AE, Hogikyan ND, Feldman EL. Disorders of cranial nerves IX and X. Semin Neurol. 2009;29:85-92.

7. Rea P. Essential clinically applied anatomy of the peripheral nervous system in the head and neck. Amsterdam: Elsevier Inc.; 2016.

8. Daniels SK. Dysphagia following stroke. 2nd ed. San Diego: Plural Publishing Inc.; 2014.

9. Shah JP, Patel SG, Singh B, Wong R. Jatin Shah's head and neck surgery and oncology. 5th ed. New York: Elsevier; 2019.

10. Hatton KW, McLarney JT, Pittman T, Fahy BG. Vagal nerve stimulation: overview and implications for anesthesiologists. Anesth Analg. 2006;103:1241-9.

11. Martinez JM, Marangell LB, Hollrah L. Vagus nerve stimulation: current use and potential applications in child and adolescent psychiatry. Child Adolesc Psychiatr Clin N Am. 2005;14:177-91.

12. Parhizgar F, Nugent K, Raj R. Obstructive sleep apnea and respiratory complications associated with vagus nerve stimulators. J Clin Sleep Med. 2011;7:401-7.

13. Morris GL III, Gloss D, Buchhalter J, Mack KJ, Nickels K, Harden C. Evidence-based guideline update: vagus nerve stimulation for the treatment of epilepsy: report of the guideline development subcommittee of the american academy of neurology. Epilepsy Curr. 2013;13:297-303.

14. Ben-Menachem E. Vagus-nerve stimulation for the treatment of epilepsy. Lancet Neurol. 2002;1:477-82.

15. Elliott RE, Morsi A, Kalhorn SP, Marcus J, Sellin J, Kang M, Silverberg A, Rivera E, Geller E, Carlson C, Devinsky O, Doyle WK. Vagus nerve stimulation in 436 consecutive patients with treatment-resistant epilepsy: long-term outcomes and predictors of response. Epilepsy Behav. 2011;20:57-63.

16. Schachter SC. Vagus nerve stimulation therapy summary: five years after FDA approval. Neurology. 2002;59:S15-20.

17. Kabir SM, Rajaraman C, Rittey C, Zaki HS, Kemeny AA, McMullan J. Vagus nerve stimulation in children with intractable epilepsy: indications, complications and outcome. Childs Nerv Syst. 2009;25:1097-100.

18. Milby AH, Halpern CH, Baltuch GH. Vagus nerve stimulation in the treatment of refractory epilepsy. Neurotherapeutics. 2009;6:228-37. 
19. Binnie CD. Vagus nerve stimulation for epilepsy: a review. Seizure. 2000;9:161-9.

20. Berry SM, Broglio K, Bunker M, Jayewardene A, Olin B, Rush AJ. A patient-level meta-analysis of studies evaluating vagus nerve stimulation therapy for treatment-resistant depression. Med Devices (Auckl). 2013;6:17-35.

21. Heijnen BJ, Speyer R, Baijens LW, Bogaardt HC. Neuromuscular electrical stimulation versus traditional therapy in patients with Parkinson's disease and oropharyngeal dysphagia: effects on quality of life. Dysphagia. 2012;27:336-45.

22. Kraaijenga SA, van der Molen L, van den Brekel MW, Hilgers FJ. Current assessment and treatment strategies of dysphagia in head and neck cancer patients: a systematic review of the 2012/13 literature. Curr Opin Support Palliat Care. 2014;8:152-63.

23. Campbell-Taylor I. Oropharyngeal dysphagia in long-term care: misperceptions of treatment efficacy. J Am Med Dir Assoc. 2008;9:523-31.

24. Baijens LW, Speyer R. Effects of therapy for dysphagia in Parkinson's disease: systematic review. Dysphagia. 2009;24:91-102.

25. Collaboration TC. Cochrane handbook for systematic Reviews of Interventions Version 5.1.0. In: Higgins J, Green S (eds.). Chapter 8: Assessing risk of bias in included studies. 2011.

26. Moher D, Shamseer L, Clarke M, Ghersi D, Liberati A, Petticrew M, Shekelle P, Stewart LA, Group P-P. Preferred reporting items for systematic review and meta-analysis protocols (PRISMA-P) 2015 statement. Syst Rev. 2015;4:1.

27. Siwek J, Gourlay ML, Slawson DC, Shaughnessy AF. How to write an evidence-based clinical review article. Am Fam Physician. 2002;65:251-8.

28. (FDA) USFaDA. National adverse drug reaction directory; COSTART (coding symbols for thesaurus of adverse reaction terms). Rockville, 1970.

29. Lundgren J, Ekberg O, Olsson R. Aspiration: a potential complication to vagus nerve stimulation. Epilepsia. 1998;39:998-1000.

30. Schallert G, Foster J, Lindquist N, Murphy JV. Chronic stimulation of the left vagal nerve in children: effect on swallowing. Epilepsia. 1998;39:1113-4.

31. Zalvan C, Sulica L, Wolf S, Cohen J, Gonzalez-Yanes O, Blitzer A. Laryngopharyngeal dysfunction from the implant vagal nerve stimulator. Laryngoscope. 2003;113:221-5.

32. Marrosu F, Maleci A, Cocco E, Puligheddu M, Barberini L, Marrosu MG. Vagal nerve stimulation improves cerebellar tremor and dysphagia in multiple sclerosis. Mult Scler. 2007;13:1200-2.

33. Barkmeier JM, Bielamowicz S, Takeda N, Ludlow CL. Modulation of laryngeal responses to superior laryngeal nerve stimulation by volitional swallowing in awake humans. J Neurophysiol. 2000;83:1264-72.

34. Broniatowski M, Grundfest-Broniatowski S, Tyler DJ, Scolieri P, Abbass F, Tucker HM, Brodsky S. Dynamic laryngotracheal closure for aspiration: a preliminary report. Laryngoscope. 2001;111:2032-40.

35. Broniatowski M, Moore NZ, Grundfest-Broniatowski S, Tucker HM, Lancaster E, Krival K, Hadley AJ, Tyler DJ. Paced glottic closure for controlling aspiration pneumonia in patients with neurologic deficits of various causes. Ann Otol Rhinol Laryngol. 2010;119:141-9.

36. Bowen AJ, Nowacki AS, Kominsky AH, Trask DK, Benninger MS, Bryson PC. Voice and swallowing outcomes following hypoglossal nerve stimulation for obstructive sleep apnea. Am J Otolaryngol. 2018;39:122-6.

37. Dawson J, Pierce D, Dixit A, Kimberley TJ, Robertson M, Tarver B, Hilmi O, McLean J, Forbes K, Kilgard MP, Rennaker RL, Cramer SC, Walters M, Engineer N. Safety, feasibility, and efficacy of vagus nerve stimulation paired with upper-limb rehabilitation after ischemic stroke. Stroke. 2016;47:143-50.
38. Aaronson ST, Carpenter LL, Conway CR, Reimherr FW, Lisanby SH, Schwartz TL, Moreno FA, Dunner DL, Lesem MD, Thompson PM, Husain M, Vine CJ, Banov MD, Bernstein LP, Lehman RB, Brannon GE, Keepers GA, O'Reardon JP, Rudolph RL, Bunker M. Vagus nerve stimulation therapy randomized to different amounts of electrical charge for treatment-resistant depression: acute and chronic effects. Brain Stimul. 2013;6:631-40.

39. Rush AJ, Marangell LB, Sackeim HA, George MS, Brannan SK, Davis SM, Howland R, Kling MA, Rittberg BR, Burke WJ, Rapaport MH, Zajecka J, Nierenberg AA, Husain MM, Ginsberg D, Cooke RG. Vagus nerve stimulation for treatment-resistant depression: a randomized, controlled acute phase trial. Biol Psychiatry. 2005;58:347-54.

40. Rush AJ, Sackeim HA, Marangell LB, George MS, Brannan SK, Davis SM, Lavori P, Howland R, Kling MA, Rittberg B, Carpenter L, Ninan P, Moreno F, Schwartz T, Conway C, Burke M, Barry JJ. Effects of 12 months of vagus nerve stimulation in treatment-resistant depression: a naturalistic study. Biol Psychiatry. 2005;58:355-63.

41. Schlaepfer TE, Frick C, Zobel A, Maier W, Heuser I, Bajbouj M, O'Keane V, Corcoran C, Adolfsson R, Trimble M, Rau H, Hoff HJ, Padberg F, Muller-Siecheneder F, Audenaert K, Van den Abbeele D, Stanga Z, Hasdemir M. Vagus nerve stimulation for depression: efficacy and safety in a European study. Psychol Med. 2008;38:651-61.

42. Rush AJ, George MS, Sackeim HA, Marangell LB, Husain MM, Giller C, Nahas Z, Haines S, Simpson RK Jr, Goodman R. Vagus nerve stimulation (VNS) for treatment-resistant depressions: a multicenter study. Biol Psychiatry. 2000;47:276-86.

43. Sackeim HA, Rush AJ, George MS, Marangell LB, Husain MM, Nahas Z, Johnson CR, Seidman S, Giller C, Haines S, Simpson RK Jr, Goodman RR. Vagus nerve stimulation (VNS) for treatment-resistant depression: efficacy, side effects, and predictors of outcome. Neuropsychopharmacology. 2001;25:713-28.

44. Lundgren J, Amark P, Blennow G, Stromblad LG, Wallstedt L. Vagus nerve stimulation in 16 children with refractory epilepsy. Epilepsia. 1998;39:809-13.

45. Kirse DJ, Werle AH, Murphy JV, Eyen TP, Bruegger DE, Hornig GW, Torkelson RD. Vagus nerve stimulator implantation in children. Arch Otolaryngol Head Neck Surg. 2002;128:1263-8.

46. Ryzi M, Brazdil M, Novak Z, Chrastina J, Oslejskova H, Rektor I, Kuba R. Long-term vagus nerve stimulation in children with focal epilepsy. Acta Neurol Scand. 2013;127:316-22.

47. Pearl PL, Conry JA, Yaun A, Taylor JL, Heffron AM, Sigman M, Tsuchida TN, Elling NJ, Bruce DA, Gaillard WD. Misidentification of vagus nerve stimulator for intravenous access and other major adverse events. Pediatr Neurol. 2008;38:248-51.

48. Lange G, Janal MN, Maniker A, Fitzgibbons J, Fobler M, Cook D, Natelson BH. Safety and efficacy of vagus nerve stimulation in fibromyalgia: a phase I/II proof of concept trial. Pain Med. 2011;12:1406-13.

49. Scherrmann J, Hoppe C, Kral T, Schramm J, Elger CE. Vagus nerve stimulation: clinical experience in a large patient series. J Clin Neurophysiol. 2001;18:408-14.

50. McLachlan RS, Sadler M, Pillay N, Guberman A, Jones M, Wiebe S, Schneiderman J. Quality of life after vagus nerve stimulation for intractable epilepsy: is seizure control the only contributing factor? Eur Neurol. 2003;50:16-9.

51. Cristancho P, Cristancho MA, Baltuch GH, Thase ME, O'Reardon JP. Effectiveness and safety of vagus nerve stimulation for severe treatment-resistant major depression in clinical practice after FDA approval: outcomes at 1 year. J Clin Psychiatry. 2011;72:1376-82.

52. Smyth MD, Tubbs RS, Bebin EM, Grabb PA, Blount JP. Complications of chronic vagus nerve stimulation for epilepsy in children. J Neurosurg. 2003;99:500-3. 
53. Ching J, Khan S, White P, Reed J, Ramnarine D, Sieradzan K, Sandeman D. Long-term effectiveness and tolerability of vagal nerve stimulation in adults with intractable epilepsy: a retrospective analysis of 100 patients. Br J Neurosurg. 2013;27:228-34.

54. Majkowska-Zwolinska B, Zwolinski P, Roszkowski M, Drabik $\mathrm{K}$. Long-term results of vagus nerve stimulation in children and adolescents with drug-resistant epilepsy. Childs Nerv Syst. 2012;28:621-8.

55. Oliveira T, Francisco AN, Demartini ZJ, Stebel SL. The role of vagus nerve stimulation in refractory epilepsy. Arq Neuropsiquiatr. 2017;75:657-66.

56. Martin JL, Martin-Sanchez E. Systematic review and metaanalysis of vagus nerve stimulation in the treatment of depression: variable results based on study designs. Eur Psychiatry. 2012;27:147-55.

57. Handforth A, DeGiorgio CM, Schachter SC, Uthman BM, Naritoku DK, Tecoma ES, Henry TR, Collins SD, Vaughn BV, Gilmartin RC, Labar DR, Morris GL III, Salinsky MC, Osorio I, Ristanovic RK, Labiner DM, Jones JC, Murphy JV, Ney GC, Wheless JW. Vagus nerve stimulation therapy for partialonset seizures: a randomized active-control trial. Neurology. 1998;51:48-55.

58. Edwards CA, Kouzani A, Lee KH, Ross EK. Neurostimulation devices for the treatment of neurologic disorders. Mayo Clin Proc. 2017;92:1427-44.

59. Nichols JA, Nichols AR, Smirnakis SM, Engineer ND, Kilgard MP, Atzori M. Vagus nerve stimulation modulates cortical synchrony and excitability through the activation of muscarinic receptors. Neuroscience. 2011;189:207-14.

60. Hays SA, Rennaker RL, Kilgard MP. Targeting plasticity with vagus nerve stimulation to treat neurological disease. Prog Brain Res. 2013;207:275-99.

61. Porter BA, Khodaparast N, Fayyaz T, Cheung RJ, Ahmed SS, Vrana WA, Rennaker RL 2nd, Kilgard MP. Repeatedly pairing vagus nerve stimulation with a movement reorganizes primary motor cortex. Cereb Cortex. 2012;22:2365-74.

62. Meyers EC, Solorzano BR, James J, Ganzer PD, Lai ES, Rennaker RL 2nd, Kilgard MP, Hays SA. Vagus nerve stimulation enhances stable plasticity and generalization of stroke recovery. Stroke. 2018;49:710-7.

63. Ganzer PD, Darrow MJ, Meyers EC, Solorzano BR, Ruiz AD, Robertson NM, Adcock KS, James JT, Jeong HS, Becker AM,
Goldberg MP, Pruitt DT, Hays SA, Kilgard MP, Rennaker RL. Closed-loop neuromodulation restores network connectivity and motor control after spinal cord injury. Elife. 2018;7:e32058.

64. Morrison RA, Hulsey DR, Adcock KS, Rennaker RL 2nd, Kilgard MP, Hays SA. Vagus nerve stimulation intensity influences motor cortex plasticity. Brain Stimul. 2019;12:256-62.

65. Groves DA, Brown VJ. Vagal nerve stimulation: a review of its applications and potential mechanisms that mediate its clinical effects. Neurosci Biobehav Rev. 2005;29:493-500.

66. Lin HC, Barkhaus PE. Cranial nerve XII: the hypoglossal nerve. Semin Neurol. 2009;29:45-52.

67. Hadley AJ, Kolb I, Tyler DJ. Laryngeal elevation by selective stimulation of the hypoglossal nerve. J Neural Eng. 2013;10:046013.

68. Jafari S, Prince RA, Kim DY, Paydarfar D. Sensory regulation of swallowing and airway protection: a role for the internal superior laryngeal nerve in humans. J Physiol. 2003;550:287-304.

69. Miller AJ. Characteristics of the swallowing reflex induced by peripheral nerve and brain stem stimulation. Exp Neurol. 1972;34:210-22.

Publisher's Note Springer Nature remains neutral with regard to jurisdictional claims in published maps and institutional affiliations.

Michelle G. M. H. Florie MD

Walmari Pilz MSc, $\mathrm{PhD}$

Remco H. Dijkman MD

Bernd Kremer MD, PhD

Anke Wiersma MD

Bjorn Winkens $\mathrm{PhD}$

Laura W. J. Baijens MD, PhD 